Check for updates

Cite this: RSC Adv., 2019, 9, 17183

Received 12th April 2019

Accepted 28th May 2019

DOI: $10.1039 / c 9 r a 02767 j$

rsc.li/rsc-advances

\section{Self-assembled microrings of Au nanoparticle and Au nanorod clusters formed at the equators of Janus particles $\dagger$}

\author{
Yutaro Hirai (D) and Hiroshi Yabu (iD * \\ A method for fabricating polymer Janus particles with microring structures at their equators has been \\ developed. This method allows gold nanoparticles and nanorods to be aligned and densely packed along \\ the microrings.
}

The recent development of plasmonic nanomaterials has revealed the interaction between metallic nanostructures and light. Microring structures composed of metallic nanoparticles (NPs) are particularly interesting since they exhibit a strong electromagnetic reaction due to plasmonic coupling among neighboring NPs and the plasmon polariton resonance of the microring structure. ${ }^{1,2}$ This is important for realizing optical metamaterials, and microring arrays of metallic NPs on 2D substrates have been fabricated using state-of-art nanoscale lithographic techniques. ${ }^{3,4}$ However, it is still challenging to produce $3 \mathrm{D}$ arrays of microring structures comprising metallic NPs.

Some approaches have been reported to successfully produce 3D metal NP clusters in the form of colloidal metamaterials, or so-called "metafluids". The production of 3D metal NP clusters has been achieved by controlling the electrostatic and hydrophobic interactions among NPs. ${ }^{5-7}$ Manna and co-workers studied optical magnetism in silver (Ag) NP clusters prepared by assembling the NPs on thiol-terminated silica particles. ${ }^{8}$ Formation of Au NP cluster shells on coreshell particles with amino-terminated polybutadiene shells and polystyrene cores has also been reported; strong plasmonic coupling and near infrared (NIR) absorption was observed. ${ }^{9}$ Thus, three-dimensionally symmetric metal NP clusters have been successfully prepared on spherical particles. However, fabrication of metal microring structures comprising metal NPs, which have intrinsically asymmetric structures, has not been realized yet in colloidal materials.

Recent developments in polymer particle preparation techniques has allowed the formation of various nanostructured particles including patchy, ${ }^{\mathbf{1 0}}$ striped, ${ }^{\mathbf{1 1}}$ core-shell ${ }^{\mathbf{1 2}}$ and Janus structures. ${ }^{13,14}$ In particular, Janus polymer colloids have attracted considerable attention due to their potential as

WPI-Advanced Institute for Materials Research (AIMR), Tohoku University, 2-1-1, Katahira, Aoba-Ku, Sendai 980-8577, Japan. E-mail: hiroshi.yabu.d5@tohoku.ac.jp $\dagger$ Electronic supplementary information (ESI) available: TG of polymer ligands and STEM image of Au NRs in Janus particles before annealing. See DOI: 10.1039/c9ra02767j pigments in electronic paper, ${ }^{\mathbf{1 5}}$ optical switches, ${ }^{\mathbf{1 6}}$ anisotropic micrometers ${ }^{17}$ and other applications. ${ }^{18}$ Recently, we have developed a method for fabricating Janus and core-shell particles by simple evaporation of a volatile organic solvent from a polymer blend solution containing water as a poor solvent. ${ }^{19}$ After solvent evaporation, the solubility of the polymer decreases with increasing content of the poor solvent, and the polymer eventually precipitates in the form of spherical particles containing two separated phases. When the interfacial tension between the two blended polymers is balanced, Janus type phase separated particles are selectively obtained using this self-organizing precipitation (SORP) process. ${ }^{20}$

In previous work, polymer stabilized inorganic NPs were incorporated into such Janus particles. ${ }^{21,22}$ Janus type phase separated particles have an interface between the two polymers and water. A three-phase contact line is formed at the equator of the particles, leading to energetic instability. If suitable nanoparticles were incorporated at the equator, they could be aligned at the interface and act as compatibilizers between the water and the two polymer domains.

Here, we report the preparation of $\mathrm{Au}$ NPs and nanorods ( $\mathrm{Au}$ $\mathrm{NRs}$ ) covered with amino-terminated polystyrene $\left(\mathrm{PS}-\mathrm{NH}_{2}\right)$ and amino-terminated polybutadiene $\left(\mathrm{PB}-\mathrm{NH}_{2}\right)$ prepared by ligand exchange. These are incorporated into $\mathrm{PS} / \mathrm{PB}$ polymer blended particles having a Janus type phase separated structure prepared by SORP. The effect of Au NP surface ligands on the composite particles is discussed.

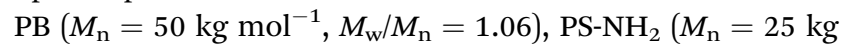
$\left.\mathrm{mol}^{-1}, M_{\mathrm{w}} / M_{\mathrm{n}}=1.04\right)$ and PB-NH $2\left(M_{\mathrm{n}}=41.5 \mathrm{~kg} \mathrm{~mol}^{-1}, M_{\mathrm{w}} / M_{\mathrm{n}}\right.$ $=1.4$ ) were purchased from Polymer Source Inc. Co. Ltd. (Montreal, Canada). PS $\left(M_{\mathrm{w}}=29.3 \mathrm{~kg} \mathrm{~mol}^{-1}\right)$ was purchased from Aldrich, United States. Tetrahydrofuran (THF/GR) was purchased from WAKO Chemical Industries, Co. Ltd., Japan. An aqueous dispersion of Au NPs (diameter $=20 \mathrm{~nm}$ ) was purchased from BBI solutions, Inc. An aqueous dispersion of $\mathrm{Au}$ NRs (short axis $=25 \mathrm{~nm}$, long axis $=100 \mathrm{~nm}$ ) was synthesized using a method reported in the literature. ${ }^{23}$ 
The fabrication process for polymer-stabilized Au NPs, Au NRs and composite Janus particles is shown in Scheme 1. PS$\mathrm{NH}_{2}$ or PB-NH $\mathrm{N}_{2}$ was dissolved in THF to prepare a $10 \mathrm{mg} \mathrm{mL}$ solution. The same amounts of the respective solutions were mixed to prepare PS- $\mathrm{NH}_{2}$ and $\mathrm{PB}-\mathrm{NH}_{2}$ mixed solutions. $5 \mathrm{~mL}$ of an aqueous dispersion of Au NPs was mixed with $5 \mathrm{~mL}$ of PS$\mathrm{NH}_{2}, \mathrm{~PB}-\mathrm{NH}_{2}$, and PS-NH$/ 2 / \mathrm{PB}-\mathrm{NH}_{2}$ solutions, and the mixed solution was sonicated for 5 min to exchange the ligands from citrate to polymers. Saturated $\mathrm{NaCl}$ aq. was mixed into the opaque dispersion after sonication, and the THF dispersion of ligand-exchanged $\mathrm{Au}$ NPs was then separated from the water phase. Polymer-stabilized Au NPs were collected from the THF phase by centrifugation (12 $000 \mathrm{rpm}, 15 \mathrm{~min}$ ) and dried at room temperature. Polymer-stabilized Au NRs were also prepared using the same procedure.

UV-Vis spectra of the THF dispersions of Au NPs and Au NRs were measured using a UV-Vis-NIR spectrometer (V-760DS, Jasco, Japan). Fourier transform infrared (FT-IR) spectra of dried Au NPs and Au NRs were measured using a FT-IR spectrometer (FT/IR-6100, Jasco, Japan) equipped with an attenuated total reflection (ATR) unit. Thermogravimetric (TG) analysis was performed using Thermo plus Evo2, RIGAKU, Japan. The shapes of the NPs and NRs were observed using transmission electron microscopy (TEM, H-7650, Hitachi, Japan).

$0.5 \mathrm{~mL}$ of THF solutions of PS and PB were mixed with $0.1 \mathrm{~mL}$ of a THF dispersion of Au NPs whose optical density was 0.8 at $\lambda=530 \mathrm{~nm} .1 \mathrm{~mL}$ of membrane filtered water was added to the mixed THF dispersion in a glass bottle at a rate of 1 $\mathrm{mL} \min ^{-1}$ with stirring. After stirring was stopped, the THF was evaporated by immersing the bottle in a water bath at $40{ }^{\circ} \mathrm{C}$ for $12 \mathrm{~h}$. The aqueous dispersion of composite particles was annealed at $100{ }^{\circ} \mathrm{C}$ for $1 \mathrm{~h}$ in a microwave heater (Analytic Jena, Germany). The interior structure of the composite particles was observed using TEM after staining the PB moieties with $\mathrm{OsO}_{4}$. For sample preparation, $0.1 \mathrm{~mL}$ of $2 \mathrm{wt} \% \mathrm{OsO}_{4}$ aq. was added to $1 \mathrm{~mL}$ of dispersion and allowed to stand for $2 \mathrm{~h}$. After staining, the composite particles were collected by centrifugation (12 $000 \mathrm{rpm}, 15 \mathrm{~min}$ ). The sample was washed using membrane filtered water ( 3 cycles of washing and centrifugation) and finally, one drop of the aqueous dispersion of stained composite particles was placed on a $\mathrm{Cu}$ grid with an elastic carbon membrane. After drying at room temperature, the interior structure of the composite particles was imaged using TEM.

Fig. 1(a) shows a typical TEM image of Au NPs@PS- $\mathrm{NH}_{2} / \mathrm{PB}-$ $\mathrm{NH}_{2}$. Monodisperse Au NPs covered with polymer shells can be seen. In order to confirm ligand exchange, UV-Vis and FT-IR

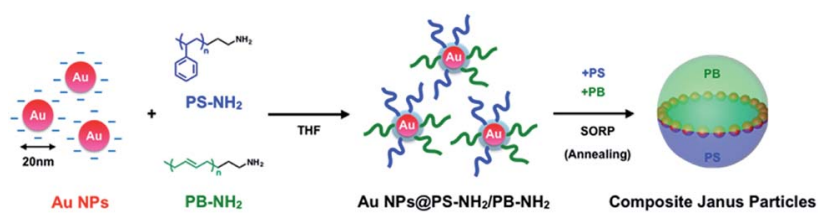

Scheme 1 Schematic illustration of preparation of polymer stabilized Au NPs and composite Janus particles.

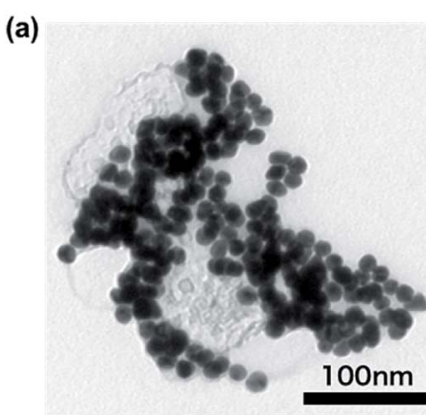

(b)

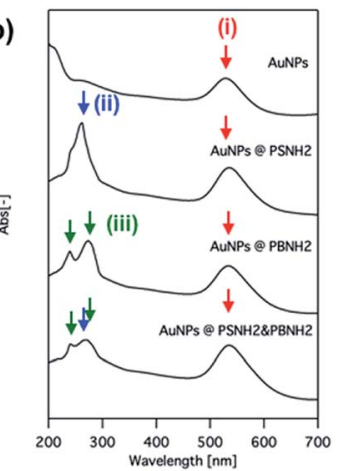

(c)

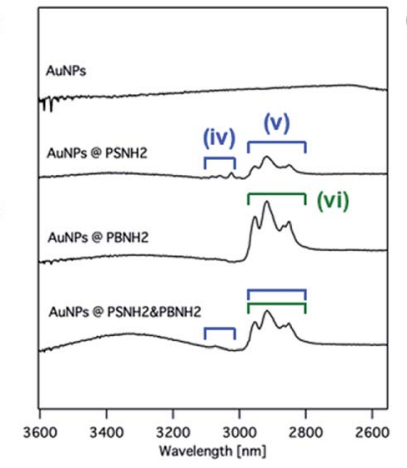

(d)

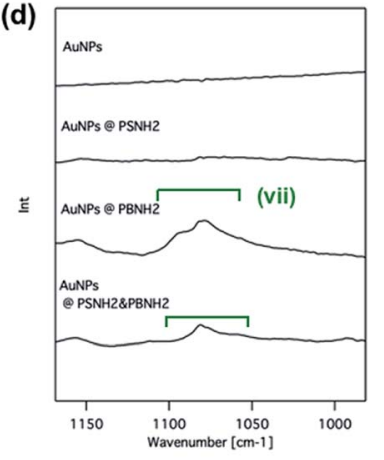

Fig. 1 TEM image of Au NPsaPS-NH $\mathrm{N}_{2} / \mathrm{PB}-\mathrm{NH}_{2}$ (a), UV-Vis spectra (b) and FT-IR spectra (c), (d) of original Au NPs, Au NPs@PS-NH $\mathrm{N}_{2}, \mathrm{Au}$ $\mathrm{NPs}$ (aPB- $\mathrm{NH}_{2}$ and Au NPs@PS- $\mathrm{NH}_{2} / \mathrm{PB}-\mathrm{NH}_{2}$, respectively.

ATR spectra of the original Au NPs, Au NPs@PS- $\mathrm{NH}_{2}, \mathrm{Au}$

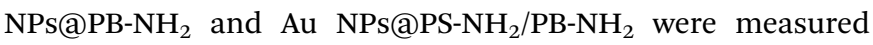
(Fig. 1(b)-(d)). In the UV-Vis absorption spectrum of the original $\mathrm{Au}$ NPs, a broad plasmonic resonance around $530 \mathrm{~nm}$ (Fig. 1(b)(i)) is clearly observed. On the other hand, absorption peaks at $260 \mathrm{~nm}$ attributed to the aromatic moiety in polystyrene (Fig. 1(b)(ii)) and two peaks attributed to double bonds of 3,4-addition and 1,2-addition in polybutadiene (Fig. 1(b)(iii)) appear. Furthermore, all of the absorption peaks present for these samples (i)-(iii) are observed in the case of Au NPs@PS$\mathrm{NH}_{2} / \mathrm{PB}-\mathrm{NH}_{2}$. These results imply that Au NPs were aminoterminated polymers selectively modified by ligand exchange. This is also confirmed by the FT-IR spectra. There are no clear absorption peaks observed for the original Au NPs, but peaks attributed to aromatic (iv), and alkyl chains (v) are seen in the case of Au NPs@ PS-NH $\mathrm{N}_{2}$, and peaks attributed to alkyl chains (iv) and double bonds (vii) are observed in the case of Au NPs@ PB- $\mathrm{NH}_{2}$ (Fig. 1(c) and (d), respectively). All of these peaks appear in the spectrum of $\mathrm{Au}$ NPs@PS- $\mathrm{NH}_{2} / \mathrm{PB}-\mathrm{NH}_{2}$. These results confirm the modification of Au NPs with amino-terminated polymers. This is also supported by the TG measurement results shown in the ESI (Fig. $\mathrm{S} 1 \dagger$ ).

To check the effect of surface polymer ligands on the distribution of Au NPs in the composite particles, Janus particles containing PS, PB and polymer-stabilized Au NPs were prepared using SORP. Fig. 2(a) shows a TEM image of a Janus particle comprising PS, PB and Au NPs@PS-NH $\mathrm{N}_{2}$. The dark gray region represents the $\mathrm{PB}$ domain and the brighter gray region represents the PS domain. The small dark spots are Au NPs and 
are visible only in the PS domain. Same as case, Au NPs@PS$\mathrm{NH}_{2}$, Au NPs located only at the PB domain of Janus particles comprised of PS, PB and Au NPs@PB- $\mathrm{NH}_{2}$. These results indicate that the location of Au NPs can be controlled by changing the surface polymer ligands, as has been previously reported. ${ }^{21}$

On the other hand, when both types of polymers were present on the surface of the Au NPs, the NPs did not disperse in the two different phases, and most of them spontaneously selfassembled in the form of a ring at the three-phase contract line among PS, PB and water (Fig. 2(c)). It is noteworthy that Au NPs seldom assemble at the interface between PS and PB in Janus particles. Russell et al. reported that metal NPs have intrinsic amphiphilic properties and stabilize interfaces between hydrophobic and hydrophilic materials. Due to the amphiphilic nature of Au NPs, they are naturally present at the surface of the Janus particles. Since both PS and PB are immobilized at the surface of the Au NPs, they have equal affinity to both phases, and the interface between PS and PB is energetically stable for them. The spacing between the Au NPs is almost constant since steric hindrance by the polymer chains on the surface prevents them from aggregating.

Based on these findings, Au NRs were also employed to form a ring structure. Fig. 3(a) shows a TEM image of Au NRs modified with PS- $\mathrm{NH}_{2}$ and $\mathrm{PB}-\mathrm{NH}_{2}$ using the same procedure as that for Au NPs. These modified Au NRs were mixed with a THF solution of PS and PB, and Janus particles were prepared by SORP. Before thermal annealing, the Au NRs were randomly located inside the Janus particles (See ESI, S2 $\dagger$ ). On the other hand, after thermal annealing, the Au NRs were aligned along the equator of the Janus particles, forming a ring structure as in the case of Au NPs.

There are two kinds of alignment of Au NRs at the interface: one along the equator (head-to-tail), and the other
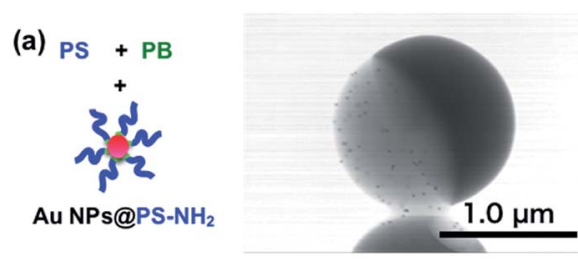

(b)

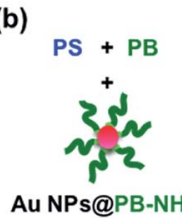

(c)

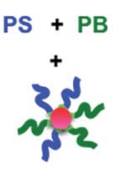

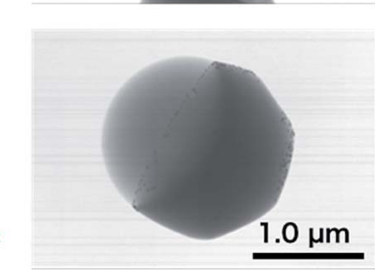

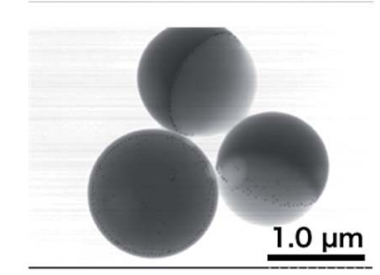

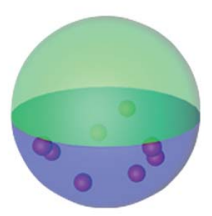
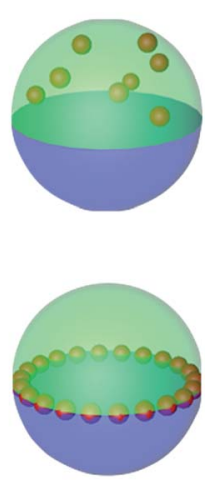

Fig. 2 Schematic illustration of mixed polymers and NPs, TEM images and model of composite Janus particles of PS/PB/Au NPs@PS- $\mathrm{NH}_{2}$ (a), $\mathrm{PS} / \mathrm{PB} / \mathrm{Au}$ NPs@PB-NH $\mathrm{H}_{2}$ (b) and PS/PB/Au NPs@PS-NH $\mathrm{N}_{2} / \mathrm{PB}-\mathrm{NH}_{2}$ (c). (a)

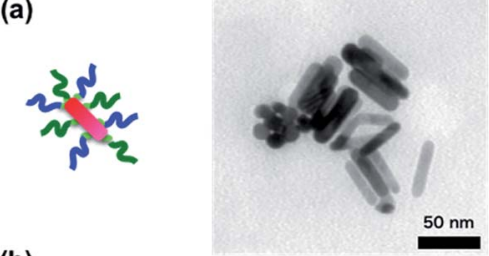

(b)
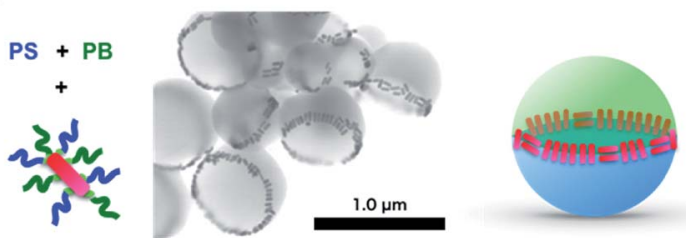

Fig. 3 Schematic illustration of mixed polymers, NPs and TEM images of $\mathrm{Au}$ NRs@PS-NH $\mathrm{H}_{2} / \mathrm{PB}-\mathrm{NH}_{2}$ (a) and PS/PB/Au NPs@PS-NH $\mathrm{H}_{2} / \mathrm{PB}-\mathrm{NH}_{2}$ after (b) annealing.

perpendicular to that (side-by-side). Most NRs are aligned sideby-side, in order to maximize the packing density at the equator. Since Au NRs have high anisotropy (aspect ratio $\sim 4$ ), four times fewer can be accommodated for the head-to-tail arrangement. Since the 1D alignment of $\mathrm{Au}$ NRs has an important effect on plasmonic resonance, ${ }^{24,25}$ controlling this alignment in a confined geometry may represent a way to tune the plasmonic properties of Au NRs.

\section{Conclusions}

In the present study, a method for fabricating microring structures at the equator of polymer Janus particles was developed. Au NPs and NRs can be aligned in a densely packed formation along the microrings. The use of surface polymer ligands allows the precise positioning of Au NPs and Au NRs without deformation of the particle shape. ${ }^{26}$ The size of the microrings can be controlled by changing the size of the Janus particles, and the optical characteristics of such microrings are expected to lead to a new class of plasmonic materials and metafluids. Additionally, since silver nanoparticles can be also modified with functionalized polymers, ${ }^{27,28}$ plasmonic absorption bands can be tuned with changing metal sources and their sizes. This strategy can be applied to a wide variety of NPs including metals and magnetic materials, leading to a large range of applications as fillers for nanocomposite materials.

\section{Conflicts of interest}

There are no conflicts to declare.

\section{Acknowledgements}

The authors thank Ms Minori Suzuki and Ms Sachie Kosaka for help with particle preparation and TEM observations. This work was supported in part by KAKENHI (17H01223, 18H05482), Ministry of Education, Culture, Sports, Science and Technology, Japan and by AMED-SENTAN (No. 16809277) grants. 


\section{Notes and references}

1 K. Bao, N. A. Mirin and P. Nordlander, Appl. Phys. A, 2010, 100, 333-339.

2 B. luk'yanchuk, N. I. Zheludev, S. A. Maier, N. J. Halas, P. Nordlander, H. Giessen and C. T. Chong, Nat. Mater., 2010, 9, 707-715.

3 W. Kubo and S. Fujikawa, Nano Lett., 2011, 11, 8-15.

4 J. Ye, F. Wen, H. Sobhani, J. B. Lassiter, P. Van Dorpe, P. Nordlander and N. J. Halas, Nano Lett., 2012, 12, 16601667.

5 A. S. Urban, X. Shen, Y. Wang, N. Large, H. Wang, M. W. Knight, P. Nordlander, H. Chen and N. J. Halas, Nano Lett., 2013, 13(9), 4399-4403.

6 N. B. Schade, M. C. Holmes-Cerfon, E. R. Chen, D. Aronzon, J. W. Collins, J. A. Fan, F. Capasso and V. N. Manoharan, Phys. Rev. Lett., 2013, 110, 148303-148306.

7 Z. Qian, S. P. Hastings, C. Li, B. Edward, C. K. McGinn, N. Engheta, Z. Fakhraai and S.-J. Park, ACS Nano, 2015, 9, 1263-1270.

8 U. Manna, J.-H. Lee, T.-S. Deng, J. Parker, N. Shepherd, Y. Weizmann and N. F. Scherer, Nano Lett., 2017, 17, 71967206.

9 M. Kanahara, H. Satoh, T. Higuchi, A. Takahara, H. Jinnai, K. Harano, S. Okada, E. Nakamura, Y. Matsuo and H. Yabu, Part. Part. Syst. Charact., 2014, 32, 441-447.

10 Z. Jin and H. Fan, Soft Matter, 2014, 10, 9212-9219.

11 J. M. Shin, Y. Kim, H. Yun, G.-R. Yi and B. J. Kim, ACS Nano, 2017, 11, 2133-2142.

12 N. Li, A. Z. Panagiotopoulos and A. Nikoubashman, Langmuir, 2017, 33, 6021-6028.

13 C. Kaewsaneha, P. Tangboriboonrat, D. Polpanich, M. Eissa and A. Elaissari, ACS Appl. Mater. Interfaces, 2013, 5(6), 18571869.
14 S. Jiang, Q. Chen, M. Tripathy, E. Luijten, K. S. Schweizer and S. Granick, Adv. Biomater., 2010, 22, 1060-1071.

15 T. Nisisako, T. Torii, T. Takahashi and Y. Takizawa, Adv. Mater., 2006, 18, 1152.

16 N. Sakamoto, Y. Hirai, T. Onodera, T. Dezawa, Y. Shibata, H. Kasai, H. Oikawa and H. Yabu, Part. Part. Syst. Charact., 2018, 36, 1800311.

17 L. Baraban, D. Makarov, O. G. Schmidt, G. Cuniberti, P. Leiderer and A. Erbe, Nanoscale, 2013, 5, 1332.

18 Y. Sheng, X. Yang, N. Yan and Y. Zhu, Soft Matter, 2013, 9, 6254-6262.

19 T. Higuchi, A. Tajima, H. Yabu and M. Shimomura, Soft Matter, 2008, 4, 1302.

20 K. Motoyoshi, A. Tajima, T. Higuchi, H. Yabu and M. Shimomura, Soft Matter, 2010, 6, 1253-1257.

$21 \mathrm{H}$. Yabu, H. Ohshima and Y. Saito, ACS Appl. Mater. Interfaces, 2014, 6, 18122-18128.

22 T. Arita, M. Kanahara, K. Motoyoshi, K. Koike, T. Higuchi and H. Yabu, J. Mater. Chem. C, 2012, 1, 207-212.

23 R. C. Wadams, L. Fabris, R. A. Vaia and K. Park, Chem. Mater., 2013, 25(23), 4772-4780.

24 D. E. Gómez, Y. Hwang, J. Lin, T. J. Davis and A. Roberts, ACS Photonics, 2017, 4, 1607-1614.

25 J. Pérez-Juste, B. Rodríguez-González, P. Mulvaney and L. M. Liz-Marzán, Adv. Funct. Mater., 2005, 15, 1065-1071.

26 V. E. Lee, C. Sosa, R. Liu, R. K. Prud'homme and R. D. Priestley, Langmuir, 2017, 33, 3444-3449.

27 Y. Saito and H. Yabu, Chem. Commun., 2015, 51(18), 37433746.

28 H. Yabu, R. Koike and Y. Hirai, J. Nanosci. Nanotechnol., 2017, 17(12), 9251-9256. 